RESEARCH ARTICLE

\title{
Design of large mode area all-solid anti-resonant fiber for high-power lasers
}

\author{
Xin Zhang ${ }^{1}$, Shoufei $\mathrm{Gao}^{2}$, Yingying Wang ${ }^{2}$, Wei Ding ${ }^{2}$, and Pu Wang ${ }^{1}$ \\ ${ }^{1}$ National Center of Laser Technology, Institute of Laser Engineering, Beijing University of Technology, Beijing 100124, China \\ ${ }^{2}$ Institute of Photonics Technology, Jinan University, Guangzhou 510632, China
}

(Received 19 August 2020; revised 16 January 2021; accepted 26 January 2021)

\begin{abstract}
High-power fiber lasers have experienced a dramatic development over the last decade. Further increasing the output power needs an upscaling of the fiber mode area, while maintaining a single-mode output. Here, we propose an all-solid anti-resonant fiber (ARF) structure, which ensures single-mode operation in broadband by resonantly coupling higherorder modes into the cladding. A series of fibers with core sizes ranging from 40 to $100 \mu \mathrm{m}$ are proposed exhibiting maximum mode area exceeding $5000 \mu \mathrm{m}^{2}$. Numerical simulations show this resonant coupling scheme provides a higher-order mode (mainly $\mathrm{TE}_{01}, \mathrm{TM}_{01}$, and $\mathrm{HE}_{21}$ ) suppression ratio of more than $20 \mathrm{~dB}$, while keeping the fundamental mode loss lower than $1 \mathrm{~dB} / \mathrm{m}$. The proposed structure also exhibits high tolerance for core index depression.
\end{abstract}

Keywords: fiber design; laser amplifiers; modeling and optimization; optical devices; optical materials

\section{Introduction}

Ultrafast fiber lasers ${ }^{[1]}$ have demonstrated outstanding performance in $\operatorname{LIDAR}^{[2]}$, spectroscopy ${ }^{[3]}$, medicine ${ }^{[4]}$ as well as material processing ${ }^{[5]}$ owing to their advantages of good beam quality, compact structure, and easy thermal management. High output power and diffraction-limited beam quality achieved by ultrafast fiber lasers make this technique one of the most remarkable and promising laser technologies existing nowadays. For example, several thousand watts average power ${ }^{[6]}$, up to $26 \mathrm{~mJ}$ pulse energy ${ }^{[7]}$, and $22 \mathrm{GW}$ peak power ${ }^{[8]}$ have been reported in recent years. Nevertheless, the progress of power scaling of fiber lasers is currently under challenge. Nonlinear effects ${ }^{[9]}$ and mode instabilities ${ }^{[10]}$ have become two main factors hindering the further development of ultrafast fiber lasers. In general, increasing the mode field area and reducing the power intensity of the core are the most effective strategies to circumvent the nonlinear effects in the optical fiber. However, fibers with large mode area (LMA) usually support multimode propagation which may lead to transverse mode instabilities (TMIs) at high average power.

Correspondence to: P. Wang, Beijing University of Technology, No. 100 Pingleyuan, Chaoyang District, Beijing 100124, China; Y. Wang, Jinan University, No. 601 West Huangpu Avenue, Guangzhou 510632, China. Email: wangpuemail@ bjut.edu.cn (P. Wang); dearyingyingwang@hotmail.com (Y. Wang)
This phenomenon would drastically degrade the emitted beam quality of the fiber laser system.

In the past few years, several LMA fibers emerged following the development of photonic crystal fibers (PCFs), allowing effective single-mode operation: large-pitch fibers ${ }^{[11]}$, all-solid photonic bandgap fibers ${ }^{[12]}$, chirally coupled core fibers $^{[13]}$, leakage channel fibers ${ }^{[14]}$, and distributed mode filtering fibers ${ }^{[15]}$. Among them, the chirally coupled core fibers and the leakage channel fibers have only achieved mode areas in the range of $1500-3500 \mu \mathrm{m}^{2}$ at $1.06 \mu \mathrm{m}^{[13,14]}$, which could not sustain a further increase of the output power of the fiber laser. The all-solid photonic bandgap fibers have achieved single-mode regime near the high-frequency end of the bandgap ${ }^{[16]}$. In this region, only a narrow spectrum range can support the low loss transmission of the fundamental mode (FM). This not only increases the difficulty of reproducible fabrication for low loss and single-mode guidance at laser wavelength, but also has a negative effect on the amplification process of ultra-fast lasers. High-power scaling capabilities have been demonstrated by using the large-pitch fibers in which single-mode operation is ensured thanks to higher-order mode (HOM) delocalization ${ }^{[11]}$. However, air clad in the cladding structure and index match between the active core and surrounding material lead to an open structure, in which core and cladding modes can interact. With the increase of the cladding diameters, at some point, 
the effective index of the cladding modes could reach that of the FM in the core, leading to anti-crossing and reduction of the laser reliability ${ }^{[17]}$.

In recent years, hollow-core $\mathrm{PCF}^{[18]}$ has undergone a tremendous development. In particular, hollow-core antiresonant fiber (ARF) ${ }^{[19]}$ has gained much interest thanks to its wide transmission bandwidth, low transmission loss, and high mode purity. Robust single-mode guidance of the fiber is achieved thanks to the resonant coupling provided by the capillaries which form the cladding structure. When the HOMs in the core phase match with the fundamental/highorder capillary modes, they could quickly leak out to the cladding. The phase matching required for resonant coupling could be achieved in broadband, independent of the absolute physical dimensions of the fiber. A larger than $20 \mathrm{~dB}$ HOM suppression ratio has been demonstrated in hollowcore $\mathrm{ARF}^{[20]}$, providing a new idea for LMA fiber to filter unwanted HOMs.

In this article, we propose an all-solid ARF structure and clarify the resonant coupling principle for this structure. Loss characteristics of FM and first HOM are explored in different core size designs. It is found that single-mode transmission can still be ensured when the core diameter is up to $100 \mu \mathrm{m}$ with the effective area larger than $5000 \mu \mathrm{m}^{2}$. The impact of core index depression on mode discrimination is also investigated.

\section{Design of all-solid anti-resonant fiber}

We propose an all-solid ARF structure shown in Figure 1(a). The light guidance of the fiber is based on the anti-resonant reflecting optical waveguide (ARROW) model ${ }^{[21]}$ and multilayered model ${ }^{[22]}$. The large core (inner diameter $D$ ) is surrounded by a single ring of non-touching capillaries in the cladding with wall thickness $t$ and inner diameter $d$. We call these non-touching capillaries anti-resonant elements (AREs). The low-refractive-index core and high-refractiveindex AREs can be considered as a Fabry-Perot-like (FPlike) resonator. Narrowband resonances of this FP resonator correspond to transmission minima for the light propagating in the core. The light will suffer high loss through leakage, when the thickness of ARE satisfies the resonance condition. The resonance wavelength is defined by $\lambda_{m}=\frac{2 t}{m} \sqrt{n_{2}^{2}-n_{1}^{2}}$, where $n_{1}$ is the refractive index of the background material, $n_{2}$ is the refractive index of ARE, $t$ is the thickness of the ARE, and $m=1,2 \ldots$ represents the order of resonance. At other wide anti-resonance wavelengths, the light is well confined in the core with low leakage loss. The high transmission coefficient originates from the anti-resonant nature of the ARE with respect to the transverse propagation constant. Other effects, such as single-path Fresnel transmission through cascaded interfaces, near-grazing incidence, and glass wall shape, play the role of further enhancing or harnessing light confinement ${ }^{[22]}$. In conclusion, concerted operations of these effects give rise to a low leakage loss in all-solid ARF.

In this work, the background material is assumed as silica with refractive index of $n_{1}=1.45$. The refractive index of ARE is set as $n_{2}=1.48$, which could be doped silica material such as germanium-doped silica ${ }^{[16]}$. Similar to the large pitch fiber and the all-solid photonic bandgap fiber ${ }^{[11,12]}$, the refractive index of the active core (assumed to be ytterbium dopant) needs to be index-matched to the surrounding background material as 1.45 . This could be done by doping the core with low-index material such as fluorine ${ }^{[23]}$. The refractive index profile of the proposed fiber is shown in Figure 1(b). The simulations were performed using a commercial finite-element method (COMSOL Multiphysics) ${ }^{[24]}$ combined with optimized mesh size and perfectly matched layer.

\section{Resonant coupling for all-solid ARF}

We determine the effective single-mode operation for the LMA fiber by setting an upper limit of the propagation losses of the FM to $1 \mathrm{~dB} / \mathrm{m}$ and a lower limit of the propagation losses of the HOMs to $10 \mathrm{~dB} / \mathrm{m}^{[25]}$. In addition, HOM suppression ratio is another effective way to judge the mode characteristics. According to the TIA-455-80-C standard $^{[26]}$, an HOM suppression ratio higher than $19.3 \mathrm{~dB}$ (a)

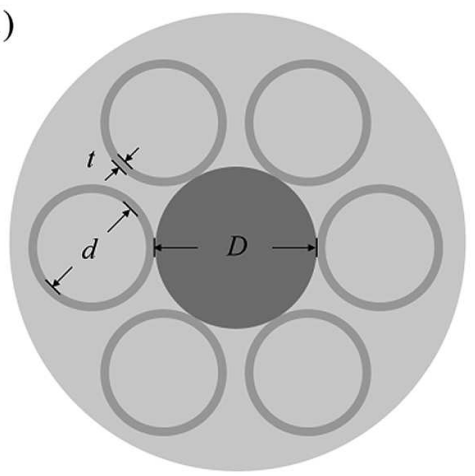

(b)

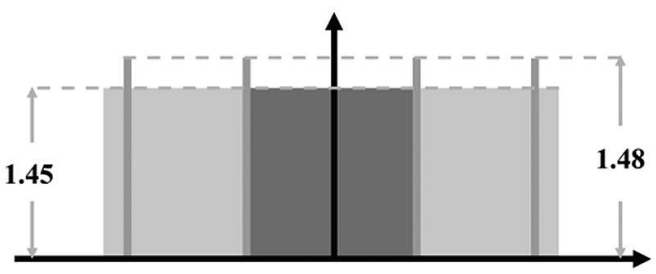

Figure 1. (a) Schematic cross-section of the proposed fiber structure. (b) Refractive index profile of the proposed optical fiber. 
is usually considered as only supporting FM in the LMA fiber. As stated before, our primary concern is the first HOM and its suppression ratio to the FM. The reason is that $\mathrm{TE}_{01}, \mathrm{TM}_{01}$, and $\mathrm{HE}_{21}$ are degenerate mode group with the lowest loss and the closest refractive index to the $\mathrm{HE}_{11}$ mode compared with other HOMs. The resonant coupling of these three modes takes place under almost the same conditions. Coupling is difficult for other HOMs because their refractive index curves are far away from that of the FM. In addition, the HOMs have higher leakage losses and lower overlap with the core ${ }^{[11]}$, making them difficult to excite in the fiber amplifier. Therefore, it is generally considered that their adverse impact on the laser system is negligible ${ }^{[25,27,28]}$.

A simple analytical model can be used to give a physical interpretation. We treat core and ARE in the cladding as two coupled capillaries. The effective index of modes guided in the capillary can be described by a modified MarcatiliSchmeltzer model ${ }^{[29]}$ :

$$
n_{\mathrm{eff}}=n_{m}-\frac{1}{2}\left(\frac{u_{n m}}{\pi \sqrt{n_{m}}}\right)^{2} \cdot\left(\frac{\lambda}{d_{\mathrm{eff}}}\right)^{2}
$$

where $n_{m}$ is the refractive index of background material in the capillary $\left(n_{m}=n_{\text {silica }}=1.45\right), u_{n m}$ is the $m$ th zero of the Bessel function $J_{n}, \lambda$ is the resonant wavelength, and $d_{\text {eff }}$ is the effective diameter, which is equal to the equivalent coefficient $\left(e_{\mathrm{c}}\right)$ multiplying inner diameter of capillary. Here $e_{\mathrm{c}}$ is used to compensate for the effects of the non-circular core and the finite wall thicknesses of the AREs. The effective indices of the first HOM equal that of ARE FM at resonant coupling wavelength. According to Equation (1), we can obtain the approximate expressions for $d / D$ :

$$
\frac{d}{D}=\frac{u_{11 \cdot \mathrm{ARE}}}{u_{01 \cdot \mathrm{core}}} \cdot \frac{e_{\mathrm{c} \cdot \mathrm{core}}}{e_{\mathrm{c} \cdot \mathrm{ARE}}} .
$$

Equation (2) gives a convenient prediction for resonance condition at which the first HOM and ARE FM couple optimally.

To analyze the influence of resonant coupling on the modal properties of all-solid ARF, we compared two fiber designs with different $d / D$ ratios. These two fibers adopted a core diameter $D=40 \mu \mathrm{m}$ and 6 capillaries in the cladding with the same wall thickness $t=1.54 \mu \mathrm{m}$. Fiber 1 was set to satisfy the condition for resonant coupling between the first HOM and ARE FM at $1.06 \mu \mathrm{m}$. Combining Equation (2) and the numerical results of finite-element simulations, we find the optimized $d / D$ value is 0.66 for Fiber 1 . Fiber 2 has a non-optimized $d / D$ ratio of 0.5 , which is used as comparison. Figure 2(b) shows the calculated effective index of the FM, first HOM of the core modes, and ARE FM as a function of

(a)

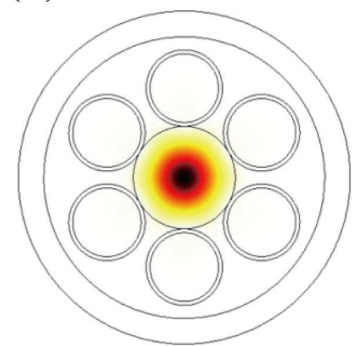

Fiber 1 - FM

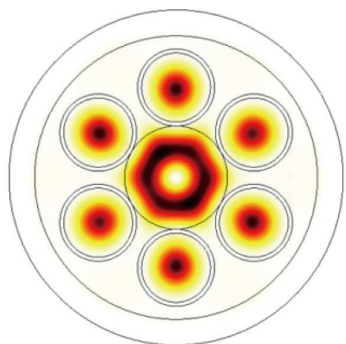

Fiber 1 - 1 $^{\text {st }}$ HOM (b)

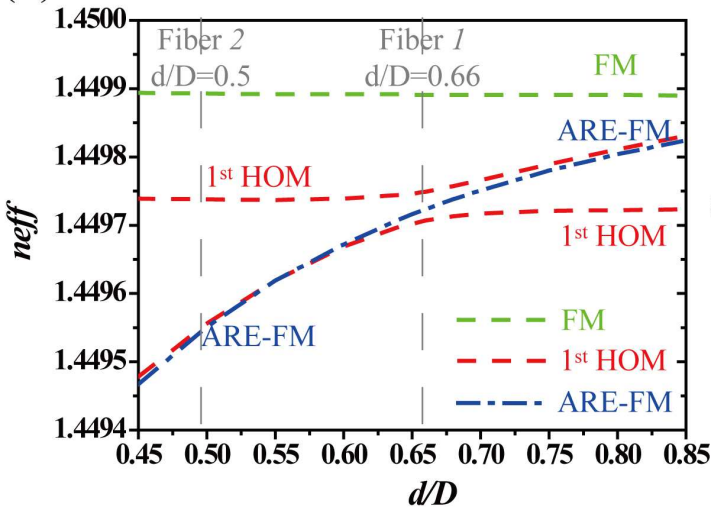

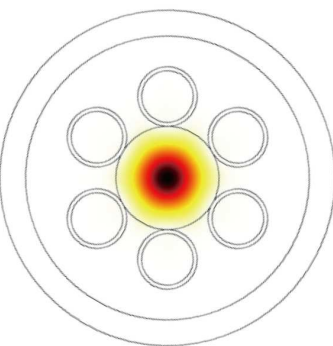

Fiber 2 - FM

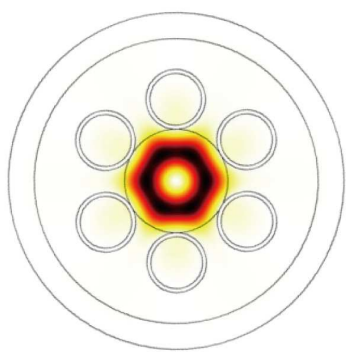

Fiber 2 - 1st $^{\text {HOM }}$

(c)

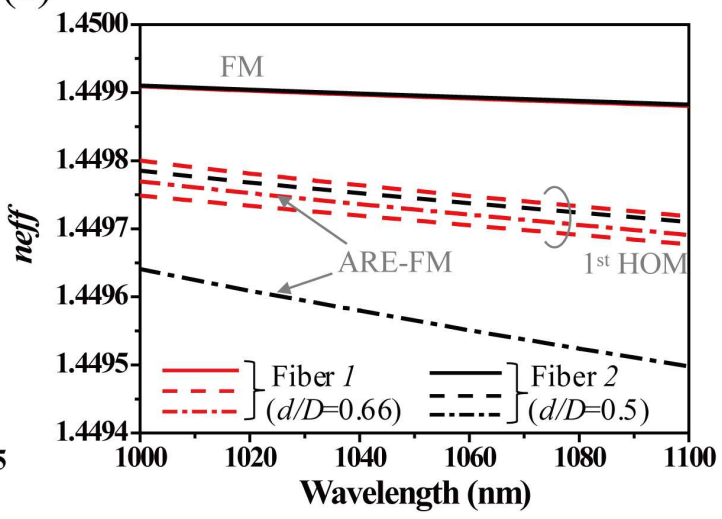

Figure 2. (a) Surface profile of the power flow of the FM and the first HOM of the two fibers at $1.06 \mu \mathrm{m}$. (b) Modal refractive indices of the FM (green), first HOM (red), and ARE FM (blue) as a function of $d / D$. (c) Modal refractive indices of the FM (solid), first HOM (dashed), and ARE FM (dashed and dotted) of Fiber 1 (red) and Fiber 2 (black) at $1 \mu \mathrm{m}$. 
(a)

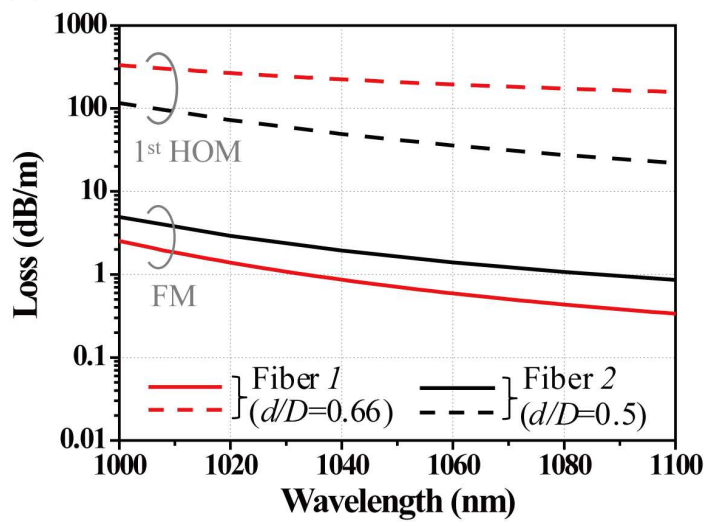

(b)

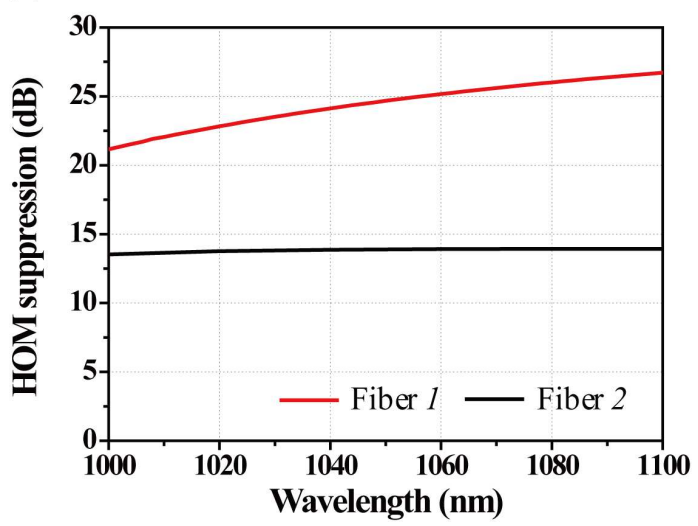

Figure 3. (a) The FM (solid) and first HOM (dashed) leakage loss for Fiber 1 (red) and Fiber 2 (black). (b) HOM suppression for the fibers in (a).

Table 1. Summary of fiber parameters for mode area scaling.

\begin{tabular}{lccccccc}
\hline $\boldsymbol{D}$ & $40 \mu \mathrm{m}$ & $50 \mu \mathrm{m}$ & $60 \mu \mathrm{m}$ & $70 \mu \mathrm{m}$ & $80 \mu \mathrm{m}$ & $90 \mu \mathrm{m}$ & $100 \mu \mathrm{m}$ \\
$\boldsymbol{d} / \boldsymbol{D}$ & 0.66 & 0.66 & 0.66 & 0.66 & 0.66 & 0.66 & 0.7 \\
$\boldsymbol{N}$ & 6 & 6 & 6 & 6 & 6 & 6 & 5 \\
\hline
\end{tabular}

$d / D$. The indices of FM for both Fiber 1 and Fiber 2 are far away from that of other modes, which can avoid the resonant coupling. In contrast, the extremely close effective index of the first HOM and ARE FM satisfies the condition of phase matching when the $d / D$ ratio is 0.66 . As a result, typical resonant coupling occurs between the first HOM, which splits the first HOM into even and odd eigenmodes, and ARE FM. For a smaller or larger $d / D$ ratio away from the phase matching point, the even and odd eigenmodes asymptotically evolve into uncoupled first HOM and ARE FM, representing the weakened resonant coupling effect. Figure 2(a) shows power flow of the FM and the first HOM of the two fibers at $1.06 \mu \mathrm{m}$. When the first HOM mode is excited in the core of Fiber 1, part of the energy is coupled to the ARE FM in the cladding, resulting in high leakage loss. Figure 2(c) indicates that the smaller index interval between the first HOM and ARE FM of Fiber 1 allows them to interact over a broadband, whereas ARE FM of Fiber 2 hardly affects the first HOM.

In Figure 3(a), we plot the loss figures of the two designed fibers. Fiber 1 has an FM loss around $1 \mathrm{~dB} / \mathrm{m}$ and an HOM loss above $100 \mathrm{~dB} / \mathrm{m}$ whereas Fiber 2 exhibits twice as high FM loss and five times lower HOM loss. This leads to an HOM suppression ratio of $22 \mathrm{~dB}$ for Fiber 1 and below $15 \mathrm{~dB}$ for Fiber 2, as a result of efficient and inefficient resonant coupling of HOM. Noticeably, a higher than $20 \mathrm{~dB}$ HOM suppression ratio over $100 \mathrm{~nm}$ bandwidth with a typical value of $25 \mathrm{~dB}$ at $1.06 \mu \mathrm{m}$ is achieved in Fiber 1, indicating it could operate in the broadband single-mode regime for many high-power ultrafast laser applications.

\section{Mode area scaling}

In the following section, the HOM suppression versus the mode field area is discussed. We increase the mode area of the fiber while keeping the thickness of ARE $t=1.54 \mu \mathrm{m}$ constant. Table 1 summarizes the parameters of the fiber studied here, where $N$ represents the number of capillaries in the cladding.

The variation of modal properties with wavelength is illustrated in Figure 4(a). In general, the leakage losses for both the FM and first HOM decrease as the core size increases. The reason is that enlarging the core size can reduce the grazing incidence angle. This would enhance the reflectivity at ARE interface and further prevent the leakage of the light ${ }^{[30]}$. It should be noted that when the core diameter of the fiber increases to $100 \mu \mathrm{m}$, the loss of the first HOM is only $0.65 \mathrm{~dB} / \mathrm{m}$ even though it experiences resonant coupling. To filter the first HOM more effectively, we adjusted the cladding structure and reduced the number of capillaries from six to five to increase the gap between them (see Figure 5). The larger gap makes the first HOM leak out of the core at a higher rate, but it also reasonably increases the FM leakage loss at the same time. These structures simulated here exhibit the following single-mode properties. First, the leakage losses of the first HOM are close to or higher than $10 \mathrm{~dB} / \mathrm{m}$, whereas the leakage losses of the FM are lower than $1 \mathrm{~dB} / \mathrm{m}$ at $1.06 \mu \mathrm{m}$. Second, resonant coupling is realized over $100 \mathrm{~nm}$ bandwidth with a higher than $20 \mathrm{~dB}$ HOM suppression ratio (as shown in Figure 4(b)). Figures 4(c) and 4(d) show how the core diameter affects the fiber mode leakage characteristics and HOM suppression ratio. It is 

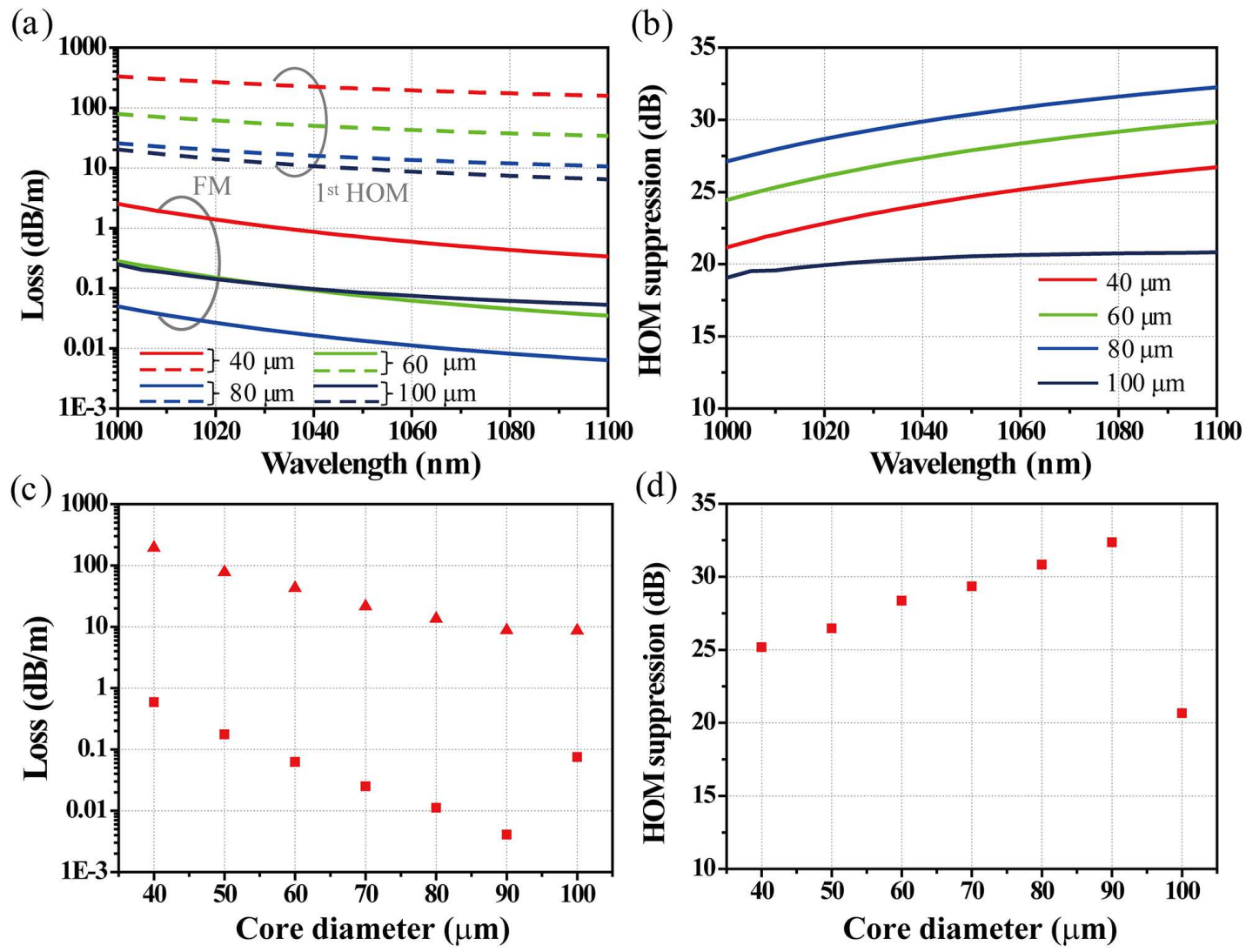

Figure 4. (a) The FM (solid) and first HOM (dashed) leakage loss under $D=40 \mu \mathrm{m}$ (red), $60 \mu \mathrm{m}$ (green), $80 \mu \mathrm{m}$ (blue), and $100 \mu \mathrm{m}$ (navy). (b) HOM suppression for the fibers in (a). (c) The FM (square) and first HOM (triangle) leakage loss with increased core diameter. (d) HOM suppression under different core sizes.
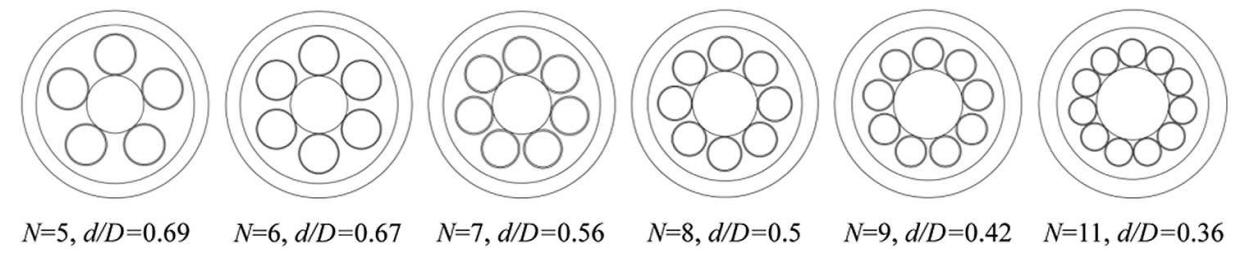

Figure 5. The legend of some structures described in Table 2 .

foreseeable that further increasing the core diameter will continuously reduce the loss of the first HOM, which will deteriorate the mode purity of the fiber. Therefore, we conclude that this all-solid ARF design could sustain a core diameter as large as $100 \mu \mathrm{m}$ and mode field area larger than $5000 \mu \mathrm{m}^{2}$.

\section{Refractive index depression of the core}

The all-solid ARF can be fabricated using the standard stackand-draw technique, which has been proved both highly flexible and capable of producing high-quality PCF of various structures ${ }^{[31]}$. The main difference of the fiber structure proposed here from others is the adding of high-refractive index ARE capillaries. This can be prepared by the modified chemical vapor deposition (MCVD) method with Ge compounds in vapor phase ${ }^{[32]}$. In the traditional MCVD method, the chemical compounds vapor periodically deposits on the inside of fused silica tubes, and then the deposited glass tube is collapsed in $\mathrm{He}$ and $\mathrm{O}_{2}$ atmosphere by increasing the burner temperature to obtain a silica glass preform. Notably, a slight change in this traditional MCVD process could produce ARE capillaries. Instead of increasing the temperature to collapse the tube, pure silica glasses can be inserted inside and outside the deposited glass tube, with negative atmosphere in between. Accordingly, glass rods containing a high refractive index ring can be produced as AREs. The subsequent production steps are similar to the method of PCF fabrication using standard stack-and-draw technique.

For our model, we assumed perfect index-matching between the active core and background material. However, achieving a perfect index matching is technologically not feasible because doping the fiber core with active ions 
Table 2. Summary of fiber parameters for refractive index depression of the core.

\begin{tabular}{|c|c|c|c|c|c|c|c|c|c|c|c|c|c|c|}
\hline \multirow[b]{2}{*}{$\Delta n$} & \multicolumn{2}{|c|}{$D=40 \mu \mathrm{m}$} & \multicolumn{2}{|c|}{$D=50 \mu \mathrm{m}$} & \multicolumn{2}{|c|}{$D=60 \mu \mathrm{m}$} & \multicolumn{2}{|c|}{$D=70 \mu \mathrm{m}$} & \multicolumn{2}{|c|}{$D=80 \mu \mathrm{m}$} & \multicolumn{2}{|c|}{$D=90 \mu \mathrm{m}$} & \multicolumn{2}{|c|}{$D=100 \mu \mathrm{m}$} \\
\hline & $\overline{d / D}$ & $N$ & $\overline{d / D}$ & $N$ & $\overline{d / D}$ & $N$ & $d / D$ & $N$ & $d / D$ & $N$ & $d / D$ & $N$ & $d / D$ & $N$ \\
\hline 0 & 0.66 & 6 & 0.66 & 6 & 0.66 & 6 & 0.66 & 6 & 0.66 & 6 & 0.66 & 6 & $\begin{array}{l}0.7 \\
\end{array}$ & 5 \\
\hline$-5 \times 10^{-5}$ & 0.6 & 7 & 0.58 & 7 & 0.56 & 7 & 0.53 & 8 & 0.5 & 8 & 0.48 & 8 & 0.46 & 8 \\
\hline$-1 \times 10^{-4}$ & 0.55 & 7 & 0.52 & 7 & 0.48 & 8 & 0.45 & 8 & 0.42 & 9 & 0.39 & 9 & 0.36 & 11 \\
\hline
\end{tabular}

(a)

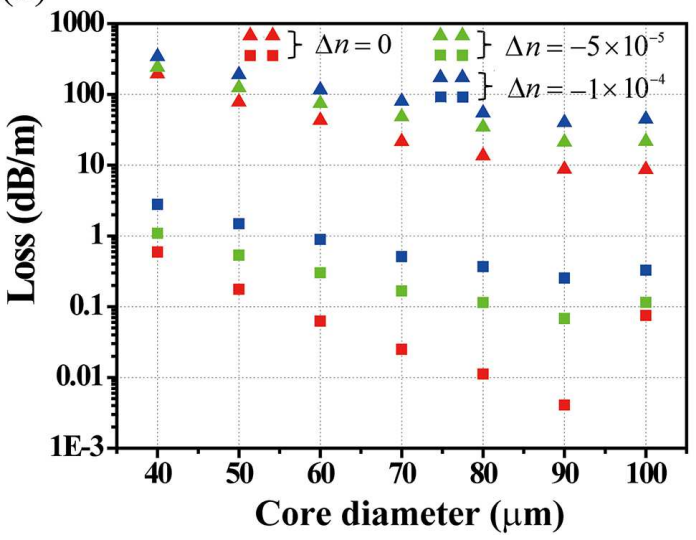

(b)

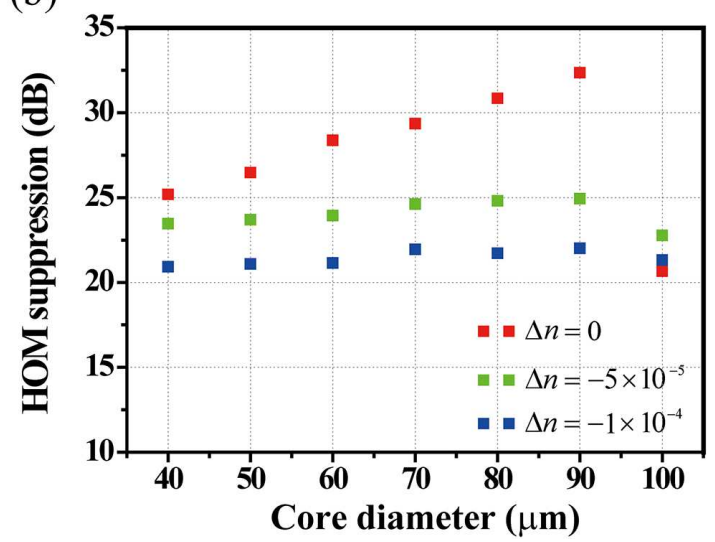

Figure 6. (a) The FM (squares) and first HOM (triangles) leakage loss for three index depression values with increasing core diameter. (b) HOM suppression for different $\Delta n$.

increases its refractive index. Therefore, co-doping with other materials (such as fluorine) is necessary to re-establish an index-matching between the core material and silica. Therefore, in our model we slightly reduced the refractive index of the core, and systematically analyzed its impact on HOM discrimination in all-solid ARF. Here $\Delta n$ is the refractive index difference between the core and the background material. The index depression of the core is set to be $\Delta n=-5 \times 10^{-5}$ and $-1 \times 10^{-4}$, respectively. It is still not easy to control the index depressions at this level, but it has been achieved in LMA fibers ${ }^{[33]}$. Index depression of the core changes the ratio of $d / D$ for resonant coupling. We used numerical simulation to find the optimal values of $d / D$ which are summarized in Table 2. Index depression enhances the leakage characteristics of the core modes and makes it easier to escape from the core. In addition, a decrease in the $d / D$ ratio increases the gap between capillaries. All of these will make the FM of the core suffer from high attenuation and affect the performance in laser applications. In order to maintain low leakage losses for the FM, the cladding structure needs to be adjusted to compensate for the influence of the index depression on the FM. Luckily, the simple and flexible ARF structure provides this degree of freedom for adjustment by increasing the number of capillaries in the cladding and reducing the gap between capillaries. Detailed structure parameters are listed in Table 2 and part of the structure is shown in Figure 5.

Figure 6(a) shows the first HOM discriminating behavior under different core index depression. When the core refractive index remains fixed, the losses of the FM and first
HOM decrease with the increase of the fiber core, which agrees with the previous discussion. Fiber with the same core size undergoes a higher leakage loss for both the FM and first HOM when the index depression increases. When the core diameter is up to $100 \mu \mathrm{m}$, the first HOM sustains $8.7 \mathrm{~dB} / \mathrm{m}, 21.6 \mathrm{~dB} / \mathrm{m}$, and $44.5 \mathrm{~dB} / \mathrm{m}$ leakage loss for $\Delta n$ of $0,-5 \times 10^{-5}$, and $-1 \times 10^{-4}$, respectively. Fortunately, the FM leakage loss is still lower than $1 \mathrm{~dB} / \mathrm{m}$ for all cases. Figure 6(b) indicates that the suppression ratio of the first HOM decreases gradually with the index depression of the core, but is still higher than $20 \mathrm{~dB}$ for different $\Delta n$. All of these show this fiber design has a good tolerance for index depression. Therefore, during the real fabrication process, we can reasonably choose the fiber core size and the fiber structure after we have the information of the core refractive index profile. This demonstrates the flexibility of the all-solid ARF structure.

\section{Discussion and conclusion}

For LMA fibers, cladding pumping is an important aspect which is critical for pump power in amplifiers. From the perspective of practical realization, the all-solid ARF can be coated with low-refractive-index polymer coating during the actual fabrication process, providing a numerical aperture for the guidance of pump light. This is the reason why we set the ARE thickness $t=1.54 \mu \mathrm{m}$, because it makes the resonant wavelength close to $915 \mathrm{~nm}$. Theoretically, at this resonant wavelength, the light will transmit freely in the whole fiber cross-section because the AREs can no longer 
effectively confine the light in the core, which will allow the pump light to be fully absorbed by the active core. The potential thermal effects in the transversal modes is another important aspect that needs to be carefully considered. In LMA fibers, the large core dimensions ensure an effective mitigation of the nonlinearities, but bring about the problem of TMIs. The thermo-optical process modifies the refractive index profile of the fiber, inducing transversally a positive quadratic reshaping of the refractive index into the gain area, which weakens the suppression effect of HOMs. Ultimately, a thermal-induced long-period grating appears in the gain area creating the conditions for fast energy transfer between the FM and the HOMs. This issue can be solved by precompensation of the core index ${ }^{[34]}$. According to simulation studies ${ }^{[34]}$, by pre-depressing the refractive index of the core, effective suppression of HOMs even under severe heat load levels can be achieved. The combination of index precompensation method and high tolerance for index depression theoretically makes such designs tolerant to TMIs.

In conclusion, we have proposed an all-solid ARF structure with large LMA and high HOM suppression. The fiber core size varies from 40 to $100 \mu \mathrm{m}$ with maximum effective mode area higher than $5000 \mu \mathrm{m}^{2}$ at $1.06 \mu \mathrm{m}$. It can achieve resonant coupling in a broadband and provide higher than $20 \mathrm{~dB}$ HOM suppression ratios. We also found that all-solid ARF has good tolerance for the index depression. Overall, these fiber designs show good potential for LMA operation in a fiber amplifier system.

\section{Acknowledgments}

This work was supported by the National Research and Development Program of China (No. 2017YFB0405200), National Natural Science Foundation of China (Nos. 61675011, 61827820, 61527822, and 61535009), Beijing Nova Program (No. Z181100006218097), Research Program of Beijing Municipal Education Commission (No. KZ201810005003), and State Key Laboratory of Advanced Optical Communication Systems and Networks, China.

\section{References}

1. M. E. Fermann, G. D. Sucha, A. Galvanauskes, M. Hofer, and D. J. Harter, Proc. SPIE 3616, 14 (1999).

2. H. Y. Xia and C. X. Zhang, Opt. Lett. 34, 2108 (2009).

3. Y. Liu, X. Zhao, B. Zhao, Z. Yao, Z. Gong, T. Y. Sui, L. Zhang, and Z. Zheng, in Conference on Lasers and Electro-Optics (2016), paper AM4K.5.

4. O. Ferhanoglu, M. Yildirim, K. Subramanian, and A. B. Yakar, in 40th Annual Northeast Bioengineering Conference (2014), p. 1.

5. T. D. Gerke, in Conference on Lasers and Electro-Optics Europe \& European Quantum Electronics Conference (2015), paper TF_1_1.
6. M. Müller, A. Klenke, A. Steinkopff, H. Stark, A. Tünnermann, and J. Limpert, Opt. Lett. 43, 6037 (2018).

7. F. Stutzki, F. Jansen, A. Liem, C. Jauregui, J. Limpert, and A. Tünnermann, Opt. Lett. 37, 1073 (2012).

8. A. Klenke, S. Hädrich, T. Eidam, J. Rothhardt, M. Kienel, S. Demmler, T. Gottschall, J. Limpert, and A. Tünnermann, Opt. Lett. 39, 6875 (2014).

9. J. Limpert, F. Röser, D. N. Schimpf, E. Seise, T. Eidam, S. Hädrich, J. Rothhardt, C. J. Misas, and A. Tünnermann, IEEE J. Sel. Top. Quantum Electron. 15, 159 (2009).

10. C. Jauregui, T. Eidam, H. J. Otto, F. Stutzki, F. Jansen, J. Limpert, and A. Tünnermann, Opt. Express 20, 12912 (2012).

11. J. Limpert, F. Stutzki, F. Jansen, H. J. Otto, T. Eidam, C. Jauregui, and A. Tünnermann, Light Sci. Appl. 1, e8 (2012).

12. L. Dong, F. T. Kong, G. C. Gu, T. W. Hawkins, M. Jones, J. Parsons, M. T. K. Dong, and K. Saitoh, IEEE J. Sel. Top. Quantum Electron. 22, 316 (2016).

13. X. Q. Ma, C. Zhu, I. N. Hu, A. Kaplan, and A. Galvanauskas, Opt. Express 22, 9206 (2014).

14. L. Dong, X. Peng, and J. Li, J. Opt. Soc. Am. B 24, 1689 (2007).

15. T. T. Alkeskjold, M. Laurila, J. Weirich, M. M. Johansen, C. B. Olausson, O. Lumholt, D. Noordegraaf, M. D. Maack, and C. Jakobsen, Nanophotonics 2, 369 (2013).

16. G. C. Gu, F. T. Kong, T. Hawkins, J. Parsons, M. Jones, C. Dunn, M. T. K. Dong, K. Saitoh, and L. Dong, Opt. Express 22, 13962 (2014).

17. F. Jansen, F. Stutzki, C. Jauregui, J. Limpert, and A. Tünnermann, Opt. Express 19, 13578 (2011).

18. J. C. Knight, Nature 424, 847 (2003).

19. W. Ding, Y. Y. Wang, S. F. Gao, M. L. Wang, and P. Wang, IEEE J. Sel. Top. Quantum Electron. 26, 4400312 (2020).

20. P. Uebel, M. C. Günendi, M. H. Frosz, G. Ahmed, N. N. Edavalath, J. M. Ménard, and P. St. J. Russell, Opt. Lett. 41, 1961 (2016).

21. N. M. Litchinitser, A. K. Abeeluck, C. Headley, and B. J. Eggleton, Opt. Lett. 27, 1592 (2002).

22. Y. Y. Wang and W. Ding, Opt. Express 25, 33122 (2017).

23. J. Limpert, O. Schmidt, J. Rothhardt, F. Röser, T. Schreiber, A. Tünnermann, S. Ermeneux, P. Yvernault, and F. Salin, Opt. Express 14, 2715 (2006).

24. S. Selleri, L. Vincetti, A. Cucinotta, and M. Zoboli, Opt. Quantum Electron. 33, 359 (2001).

25. A. Steinkopff, C. Jauregui, F. Stutzki, J. Nold, C. Hupel, N. Haarlammert, J. Bierlich, A. Tünnermann, and J. Limpert, Opt. Lett. 44, 650 (2019).

26. TIA Standards, IEC-60793-1-44 Optical Fibres - Part 1-44: Measurement Methods and Test Procedures - Cut-off Wavelength (TIA, 2020).

27. F. Stutzki, F. Jansen, C. Jauregui, J. Limpert, and A. Tünnermann, Opt. Express 19, 12081 (2011).

28. F. Jansen, F. Stutzki, H.-J. Otto, M. Baumgartl, C. Jauregui, J. Limpert, and A. Tünnermann, Opt. Express 18, 26834 (2010).

29. E. A. J. Marcatili and R. A. Schmelzter, Bell Syst. Tech. J. 43, 1783 (1964).

30. B. Debord, A. Amsanpally, M. Chafer, A. Baz, M. Maurel, J. M. Blondy, E. Hugonnot, F. Scol, L. Vincetti, F. Gérôme, and F. Benabid, Optica 4, 209 (2017).

31. P. Francesco, M. N. Petrovich, and D. J. Richardson, Nanophotonics 2, 315 (2013).

32. J. B. Macchesney, P. B. O'connor, and H. M. Presby, Proc. IEEE 62, 1280 (1974).

33. F. Jansen, F. Stutzki, H. J. Otto, T. Eidam, A. Liem, C. Jauregui, J. Limpert, and A. Tünnermann, Opt. Express 20, 3997 (2012).

34. D. Darwich, R. Dauliat, R. Jamier, A. Benoit, K. Schuster, and P. Roy, Appl. Opt. 55, 8213 (2016). 